\title{
Evaluation on Triaging Pathway Strategy Guidelines for Cases with Elipidae Bite during COVID-19 Pandemic at Emergency Department
}

\author{
Zainul Ikhwan Ahmad Khusairi \\ Emergency and Trauma Department, Taiping Hospital, Perak Darul Ridzuan, Malaysia \\ DOI: http://dx.doi.org/10.31344/ijhhs.v5i0.306
}

Introduction: Emergency departments (ED) typically practice a triage system to classify patients into priority levels. A triage system is commonly used in crowded emergency rooms to determine which patient should be seen and treated immediately.

Objective: The objective of this study was to evaluate the effectiveness of triaging pathway guidelines for critical cases seen in the Emergency Department during the COVID-19 outbreak.

Case Presentation: Emergency Department, Taiping Hospital is practicing a triaging system which consists of two divisions; cases with respiratory symptoms (SARI) and cases without symptoms of respiratory impairment (non-SARI). A patient with snake bite was presented at the Emergency Department, Taiping Hospital with symptoms of numbness at the site of the bite and a complaint of difficulty to open the eyes, while tachycardia was noted. However, no shortness of breath or difficulty in breathing was reported. COVID-19 rapid screening was done and the patient was treated under Red Zone (non-SARI). Luckily, the patient survived and was stable with no reaction observed after treated with Neuro Polyvalent Antivenom 50ml in 500ml normal saline over one hour.

Conclusion: The available patient triaging pathway strategy guideline was effectively practiced by paramedics when managing critical cases at the Emergency Department during COVID-19 outbreak. Other emergency departments are advised to practice effective and appropriate triaging pathway strategy guidelines to save patients' lives.

Keywords: triaging system, COVID-19 epidemic, critical cases, severe acute respiratory infection (SARI), emergency department 